\title{
ENFANCE, ART, MODERNITE ET POSTMODERNITE
}

\author{
Childhood, Art, Modernity and Post modernity
}

\author{
Alain KerLan \\ André D. RoberT \\ Université Lumière Lyon 2 \\ alain.kerlan@univ-lyon2.fr
}

Fecha de recepción: 03-III-2013

Fecha de aceptación: 12-III-2013

RÉSumÉ: Cet article traite de la portée relationnelle, éducative, esthétique, «démocratique» de situations qui, tendant à se généraliser dans de nombreux pays, placent de plus en plus les enfants «auprès d'artistes» à l'occasion des résidences d'artistes installées dans des écoles ou dans des centres éducatifs. Il développe une double dimension, historique et philosophique. Le sens de Modernité et Post modernité est précisé. L’approche historique rappelle les étapes de la découverte d'un sentiment de l'enfance que le Moyen Age avait laissé se dissoudre (incidemment, l'art apparaît comme un des supports de cette découverte). Critiquant les positions post modernes des nouveaux sociologues de l'enfance, l'approche philosophique révèle -dans les situations esthétiques considérées- une tension entre la relation d'égal à égal enfant/artiste (illustration possible du principe post moderne de symétrie) et le maintien de la singularité de l'artiste adulte (condition de possibilité de l'éducation, au sens moderne). L'expérience de l'enfant «auprès d'artistes" porte une intéressante possibilité de création d'une nouvelle normativité (inhérente à l'œuvre en train de se faire).

Mots-clés: Enfance, Art, Expérience d'enfants auprès d'artistes, Modernité, Post modernité.

AвsтRACT: This paper concerns the relational, educational, aesthetic, «democratic» impact of situations which, tending to become widespread in numerous countries by the world, place more and more the children «near artists» on the occasion of artists' residences settled in schools or in educational centres. The study presents a double, historical and phi- 
losophical, dimension. Modernity and post modernity meaning is determined. The historical approach calls back the stages of the discovery of «the childhood feeling» that the Middle Ages had let dissolved (by the way, the art appears as one of the supports of this discovery). Criticizing the Chidhood new sociologists post modern positions, the philosophical approach reveals -in the considered aesthetic situations- a tension between the child/artist «equal to equal» relationship (possible illustration of post modern principle of symmetry) and the preservation of the adult artist peculiarity (condition of possibility of education, in a modern sense). The «near artists» child experience brings an interesting possibility in creating a new normativity (that of art making).

Keywords: Childhood, Art, «near artists» children experience, Modernity, Post modernity.

\section{INTRODUCTION}

Les relations Enfance et Art se trouvent au centre de notre réflexion ${ }^{1}$. Il s'agit d'interroger la portée relationnelle, éducative, esthétique, "démocratique» de situations qui, tendant à se généraliser dans de nombreux pays, placent de plus en plus les enfants "auprès d'artistes» à l'occasion des résidences d'artistes installées dans des écoles ou dans des centres éducatifs. Dans cette optique, notre étude présente une double dimension, historique et philosophique. En effet, des figures de l'enfance propres à la modernité et à la postmodernité se voient possiblement mises en jeu à la lumière de ces nouveaux dispositifs.

L'approche historique rappelle les étapes de la découverte d'un sentiment de l'enfance que le Moyen Age avait laissé se dissoudre (incidemment, l'art apparaît comme un des supports de cette découverte ou redécouverte). Progressivement, de reléguée qu'elle était, l'Enfance s'impose comme la figure centrale de la famille moderne, voire de la Modernité, au point qu'on peut parler au XXe siècle d'une véritable "passionde l'enfant» qui, dramatisant le moment de l'enfance, fait de celui-ci, le porteur de tous les espoirs, le dépositaire de l'avenir, ainsi doté par les adultes d'une responsabilité extrême, en devenir. Un des derniers avatars de cet enfant moderne serait l'enfant-artiste, dont la désignation comme encore enfant indique bien le statut, à la fois valorisé, exceptionnel, temporaire (qu'adviendra-t-il de lui quand il perdra sa qualité d'enfant, au terme de son développement?) et différencié (sous la dépendance d'un étiquetage adulte). Sans doute plus interprétation de certains phénomènes à l'œuvre dans la société et l'histoire récente que réalité déjà totalement avérée, la postmodernité se présente comme une critique (à la fois en acte et théorisée par quelques philosophes et sociologues) des aspects et concepts les plus marquants, d'ailleurs encore largement en vigueur, de la modernité. Les postmodernes se

Ce travail est issu d'une recherche financée par l'ANR, Agence Nationale de la Recherche (20102013). 
montrent ainsi sensibles à «'individu éclaté, excentré, dispersé, disséminé, libre d'affirmer sa singularité contre la rigueur de tous les conformismes sociaux» ${ }^{2}$. Dans une telle perspective, ceux qu'on appelle les nouveaux sociologues de l'enfance pensent mettre au jour un changement de sens radical de l'enfance et une symétrie quasi totale avec le monde adulte, parfois à l'aide d'exemples catastrophiques.

C'est ici que, étant appelée dans notre texte à prendre le relais de l'histoire et de la sociologie, la réflexion philosophique -fondée sur des expériences d'une nature non catastrophique, mais au contraire heureuse, par le truchement de l'art- nous amène à soumettre l'hypothèse suivante: les situations d'enfants auprès d'artistes tendent à la fois à vérifier le principe postmoderne de symétrie, dans le moment artistique de la co-création enfant-artiste, et à la fois à le relativiser, tous les artistes concernés tenant à proclamer hautement leur statut et leur différence (ce que la postmodernité leur contesterait, à la manière par exemple de Joseph Beuys). C'est donc à prendre la mesure de cette tension, révélée selon nous par les situations esthétiques en question, que nous invitons le lecteur.

\section{ENFANCE, MODERNITÉ ET CRITIQUE SOCIOLOGIQUE POSTMODERNE}

\subsection{LA "DÉCOUVERTE" DE L'ENFANCE}

Comme on le sait, c'est en s'appuyant notamment sur des corpus iconiques (artistiques au sens moderne) que Philippe Ariès développa ses thèses pionnières quant à l'inexistence d'un sentiment particulier de l'enfance en Europe pendant au moins toute la période du Haut Moyen Age jusqu'au XIIe siècle ${ }^{3}$. «Enfance» est un concept ambigu, tributaire de découpages temporels variables selon les époques et les civilisations, et sous la dépendance de catégories langagières elles-mêmes différentes d'une langue à l'autre. Là où le latin distinguait sept âges (correspondant à sept planètes), et singulièrement infancia et pueritia pour caractériser les premiers temps de la vie, le français ne disposa d'abord que d'enfance pour saisir une période globale allant jusqu'à 14 ans, suivi de jeunesse (force de l'âge) et vieillesse, dans une vision à trois temps seulement. Le Grand Propriétaire de toutes choses ${ }^{4}$ de 1556 nous met cependant en présence d'un paradoxe lorsqu'il rapporte la définition latine d'infancia (coïncidant avec la période du Bas-Empire, i.e. le VI e siècle de notre ère): «Le premier âge, c'est enfance [...] commence cet âge quand l'enfant est né et dure jusqu'à sept ans, et en cet âge ce qui est né est appelé enfant,

2 D. Lecourt, Contre la peur, Paris, PUF, $4^{\mathrm{e}}$ édition, 2007, p. 129.

3 Ph. Ariès, L'enfant et la vie familiale sous l'Ancien Régime, Paris, Le Seuil, 1973, nouvelle édition.

4 Le Grand Propriétaire de toutes choses, très utile et profitable pour tenir le corps en santé, par B. de Glanville, traduit par Jean Corbichon, cité par Ariès, op. cit., p. 8. 
qui vaut autant à dire comme non parlant [...] Après enfance, vient le second âge ... on l'appelle pueritia et est ainsi appelé pour ce que en cet âge il est encore ainsi comme est la prunelle de l'œil, [...] et dure cet âge jusqu'à quatorze ans». Tout en appréciant la plus grande finesse de distinction de ce vocabulaire, nous ne pouvons que nous étonner rétrospectivement de l'application du sens littéral d'infans (celui qui ne parle pas) à des sujets pouvant avoir jusqu'à sept ans. Selon Ariès, il faut se tourner, aux alentours des XVI-XVIIe siècles, vers les classes supérieures de la société pour voir s'opérer, et/ou plus globalement vers le début du XIXe siècle pour voir se généraliser, des subdivisions plus précises (bébé, moyenne enfance, adolescence).

En tout état de cause, c'est à cette conception encore indifférenciée de l'enfance que Philippe Ariès se réfere lorsque, prétendant mettre au jour une généalogie de «la découverte de l'enfance», il établit le constat que "l'art médiéval, jusqu'au XIIe siècle environ, ne connaissait pas l'enfance ou ne tentait pas de la représenter». L'historien produit de nombreux exemples de mises en scène de corps et de visages adultes là où le titre de la miniature ou de la sculpture, ainsi que l'épisode représenté, désignent des «petits», des enfants. Il n'en a pas toujours été ainsi car, à l'époque hellénistique, l'enfant était très présent dans la statuaire, notamment sous les traits d'Eros.

La situation change avec l'apparition -à partir du XIIIe siècle- d'anges, d'abord adolescents, tel celui de la cathédrale de Reims, et pendant le Quattrocento, ceux peints par Botticelli, Ghirlandajo, Fra Angelico. Au même XIIIe siècle, «avec la maternité de la Vierge, la petite enfance pénètre dans le monde des représentations» ${ }^{6}$. Progressivement, aux siècles suivants, s'impose une image de l'enfance, non seulement sanctifiée à travers la figure du petit Jésus, mais aussi en quelque sorte sécularisée à travers la saisie des gestes et comportements enfantins les plus banals en même temps que les plus touchants. De l'enfance de Jésus à l'enfance des saints, Ariès entend repérer une multiplication des scènes d'enfants dans la peinture, la sculpture, la tapisserie, mais aussi dans la littérature à l'intention du grand public (contes et légendes des Miracles Notre-Dame par exemple). Ultérieurement (XVe-XVIe siècles) c'est à une laïcisation de l'iconographie enfantine que l'on assiste. Ainsi commence à poindre, attesté ici par les évolutions de la représentation artistique, ce que l'historien désigne sous le nom de «sentiment de l'enfance», c'est-à-dire une attitude particulièrement attentive des adultes envers l'enfant ${ }^{7}$, remplaçant une relative indifférence antérieure, et qui n'apparaît donc pas comme une donnée universelle mais bien plutôt comme un produit circonstancié de l'histoire.

Ariès, Titre du chapitre II de la première partie 3: «Le sentiment de l'enfance», op. cit., p. 23 et sq. Ariès, op. cit., p. 25.

7 In J.-B. Pontalis (dir.), L'enfant, Paris, Gallimard, Folio Essais, 1979, pp. 22-24. 
Ce sentiment s'affirme quand l'enfant peut être représenté seul («grande nouveauté du XVIIe siècle»), nu (le "putto», qui renouant avec le thème hellénistique d'Eros exprime un enfant générique) ou surtout portraituré (telle personne particulière d'enfant, représentation qui se développe au XVIIe siècle dans les tableaux des grandes familles en devenant même souvent centrale). Une reconnaissance, de même qu'un goût, de la particularité de l'enfance se donne alors progressivement à voir, qui selon Ariès avec bien d'autres phénomènes étudiés dans son livre, liés notamment aux avancées de l'institutionnalisation scolaire, d'abord dans les collèges- participe de l'avènement d'une child-oriented society, caractéristique des temps modernes. Le sentiment de l'enfance, advenu aux XVIIe-XVIIIe siècles, est double, partagé entre des comportements familiaux qui se généralisent (la pratique du "mignotage») et des réflexions intellectuellement élaborées provenant de moralistes et d'éducateurs (le souci hygiénique, moral et psychologique de l'enfant). La thèse de l'historien qui va tant marquer les esprits pendant plusieurs décennies, et garde aujourd'hui -malgré les critiques'-son prestige, est ainsi posée: la «civilisation médiévale avait oublié la paideia des anciens et elle ignorait l'éducation des modernes [...] La famille et l'école ont ensemble retiré l'enfant de la société des adultes» ${ }^{10}$.

\subsection{ENFANCE et familLe de LA MOdernité}

Cette thèse trouve sa confirmation notamment dans l'ouvrage d'Edward Shorter, The making of the Modern family ${ }^{11}$. Si l'auteur reproche à Ariès d'avoir minimisé la persistance d'un sentiment d'indifférence, et même parfois d'une attitude de véritable violence, à l'égard des enfants, dans les classes populaires jusqu'à la fin du XIXe siècle (ce qui n'est, à la lecture de L'enfant et la vie familiale, une appréciation que partiellement fondée ${ }^{12}$ ), il lui reconnaît d'avoir «été le premier à soutenir que la société traditionnelle se caractérisait par l'indifférence de la mère à l'égard du nourrisson» ${ }^{13}$. Les apports de Shorter se situent dans la même perspective, élargissant le champ d'investigation -dans un nombre conséquent de pays (Allemagne, Grande-Bretagne, France, Etats-Unis, Scandinavie) - à l'évolution de la structuration des familles, à la mutation qui fait passer de

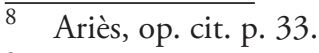

9 Par exemple contribution de J.-C. Schmitt lui-même in G. Levi, JC. Schmitt (dir.), Histoire des jeunes en Occident, Paris, Le Seuil, 1996, tome 1; voir aussi, G. Gros, «Philippe Ariès : naissance et postérité d'un modèle interprétatif de l'enfance»,Histoire de l'éducation, no 125, 2010. Op. cit. p. 462-3, et p. 465.

11 E. Shorter, Naissance de la famille moderne, XVIIIe-XXe siècle, traduction française, Paris, Le Seuil, 1977 [1975].

12 En effet on peut lire chez Ariès: «Cette indifférence [...] a persisté jusqu’au XIXe siècle, au fond des campagnes», op.cit. p. 30.

13 Shorter, op. cit. p. 210. 
la communauté traditionnelle à la "montée de la famille nucléaire» ${ }^{14}$. Trois éléments ont contribué, dans les temps modernes, à déplacer la famille de ses positions ancestralement établies: l'avènement de l'«amour romantique» qui vient contester les procédures habituelles présidant à la formation des couples; la montée de «l'esprit domestique» qui fait se replier la famille (parents-enfants) sur elle-même et advenir une préférence pour le "chez-soi» au détriment des réunions communautaires à l'extérieur, principalement masculines (mais pas seulement, concernant aussi les jeunes femmes non mariées); la modification des relations mère-enfant, ce qui confirme à nouveau les analyses d'Ariès: «Dans la société traditionnelle, bien des considérations venaient avant le bien-être du petit enfant $[. .$.$] tandis que, dans la société moderne, l'amour maternel veille à ce que le$ bien-être du nourrisson-centre du monde l'emporte sur tout autre souci» ${ }^{15}$.

Au terme d'analyses historiques très informées et détaillées traitant entre autres de ces trois éléments qui ont contribué à la naissance de la famille nucléaire moderne, Shorter conclut son étude par la formulation d'une hypothèse sur l'émergence d'une famille qu'il qualifie déjà de "postmoderne» (années 1960 et 1970). Avec l'instabilité croissante des ménages et la «destruction du nid» (c'est-à-dire l'attitude centrifuge des deux générations et sexes composant la famille nucléaire), les adolescents sont selon lui les acteurs centraux de cette nouvelle mutation: «De nos jours, le groupe des pairs de l'adolescent a repris le contrôle de son apprentissage de la vie en société [...] L'enjeu n'était pas tant l'affection que l'on portait aux parents qu'un désir d'autonomie et d'indépendance: un rejet de principe de toute tutelle parentale» ${ }^{16}$.

Concernant plus spécifiquement les enfants eux-mêmes (désormais distincts des adolescents, depuis les XVIIIe- XIXe siècles ${ }^{17}$ ), ce que pointait déjà Ariès dans la conclusion générale de son ouvrage -à savoir le développement contemporain d'orientations scientifiques dédiées à l'enfance (psychologie, psychanalyse, pédiatrie) et vulgarisées auprès du grand public, ainsi que l'obsession des «problèmes physiques, moraux, sexuels, de l'enfance» ${ }^{18}$ - s'affirme peu à peu dans toutes les classes de la société au XXe siècle, et s'amplifie à la fin de celui-ci. Dans le dernier tiers du XXe siècle en effet, à la faveur de la critique de la tradition et de l'autorité qui cristallise dans de nombreux pays développés au cours de l'année 1968, se fait jour une véritable "passion de l'enfant» ${ }^{19}$, caractérisée par des affects intenses des parents et de toute la société à l'égard de la descendance. C'est le courant psychanalytique qui a d'abord pris à sa charge scientifiquement et idéo-

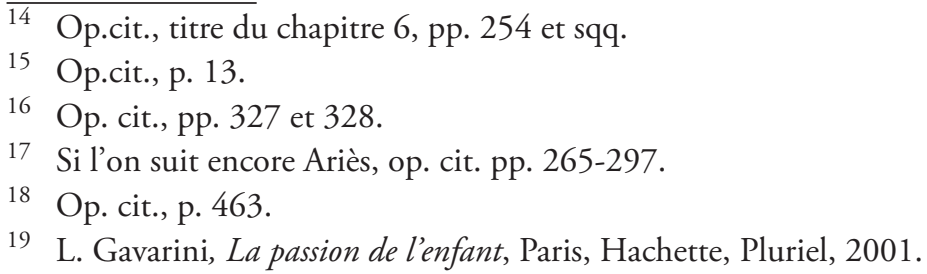


logiquement, depuis la fin de la seconde guerre mondiale, ce renouvellement de posture envers l'enfant ${ }^{20}$, jusqu'à faire advenir celui-ci comme bébé-personne et enfant sujet, doté d'une sexualité et d'un pouvoir précoce de discernement (la psychanalyste Françoise Dolto jouant de ce point de vue dans l'univers francophone, et par le truchement de sa communication à l'intention du grand public, un rôle très important). Plus près de nous, à la domination scientifique et idéologique de la psychanalyse, tend à se substituer celle de la psychologie cognitive qui promeut un enfant fondamentalement doté de compétences, sans lui dénier sa qualité de sujet.

Ce que l'on a pu appeler "passion» de l'enfant n'a donc fait que se déployer et s'accroître jusqu'à nos jours en investissant, au gré de diverses influences circonstancielles, technologiques et/ou scientifiques, de nouvelles figures (enfant maltraité, enfant victime, enfant programmé, enfant artificiellement procréé, enfant consommateur, enfant précoce, enfant acteur mais aussi hyper-protégé). Une représentation va se faire insistante: 'l'avenir est dans l'enfance'. Elle dramatise l'enfance, l'institue comme un moment décisif. Un nouveau basculement qualitatif s'est ainsi produit dans la mise au centre (de la famille, de la société) de l'enfance et des enfants, faisant de l'éducatif et du scolaire des objets d'intense angoisse des parents, et s'inscrivant dans la lignée du mouvement amorcé dès le XVIIe siècle lors de la découverte d'un sentiment de l'enfance. Pour autant, l'enfant dont il est ici question, tout en étant une personne, voire un sujet disposant de droits-libertés (expression, religion, réunion), demeure un être dont le statut reste nettement différencié de celui de l'adulte, lui offrant toujours des droits de protection (droits-créances) particuliers ${ }^{21}$.

\subsection{LA POSTMOdERNITÉ: UNE VISION AUTRE DE L'ENFANCE}

Or, certaines conceptions sociologiques opèrent aujourd'hui un nouveau déplacement dans la représentation intellectuelle de l'enfance, qui tend à changer encore singulièrement la nature de celle-ci. D'autres figures de l'enfance, certaines dramatiques, sont à ce titre parfois mises en avant: enfants travailleurs, enfants prostitués, enfants guerriers, enfants meurtriers d'autres enfants ${ }^{22}$.

Le postulat d'une égalité adulte/enfant est au principe de la «nouvelle sociologie de l'enfance» telle qu'elle se développe dans le sillage de quelqu'un comme Alan Prout. Récusant les schèmes de pensée issus de la modernité, fondés sur des dichotomies

$\overline{20}$ Cf. G. Neyrand, L'enfant, la mère et la question du père. Un bilan critique de l'évolution des savoirs sur la petite enfance, Paris, PUF, 2000.

21 Cf. Convention Internationale des Droits de l'enfant, UNICEF, 1990.

22 A., Turmel, A historical Sociology of Childhood: developmental Thinking, Categorization and Graphic Visualization. Cambridge, Cambridge University Press, 2008. 
en apparence nettement établies (nature versus culture, structure sociale versus acteurs, local versus global, devenir versus être), Alan Prout mobilise le principe dit de symétrie, emprunté à l'épistémologie de David Bloor ${ }^{23}$ et à la sociologie de Bruno Latour ${ }^{24}$, en vue de rendre compte de ce qu'il estime être l'égalité, ou la non-différenciation, des adultes et des enfants dans la société. Ainsi, pour approcher, expliquer, comprendre les enfants aujourd'hui, il faut appréhender les transformations subies par le monde au cours des vingt dernières années et ayant nécessairement affecté l'enfance. Par exemple, comment penser adéquatement l'expérience enfantine dans les vieilles catégories de la modernité, à la manière par exemple de Shorter, quand plus de $30 \%$ des familles n'étant plus des familles standard, les enfants sont amenés à «naviguer» entre plusieurs familles recomposées et à déployer ainsi des trésors de tact et de diplomatie, des compétences qui ne les mettent pas en infériorité par rapport aux adultes, tout au contraire? Quand on constate la nécessité pour les enfants de se confronter à une multiplicité de contextes et de temps éducatifs séparés, entraînant une véritable fragmentation des vécus enfantins, ce qui les oblige à construire eux-mêmes sens et cohérence (ce que déplorent les éducateurs «modernes», mais non pas les nouveaux sociologues de l'enfance $\left.{ }^{25}\right)$ ? Quand, à l'occasion du développement de nouvelles technologies, des connexions inédites se créent entre les enfants et celles-ci, inductrices d'îlots d'enfance juxtaposés et ne faisant pas spontanément de l'unitée ${ }^{26}$ Cette même sociologie relève aussi un accroissement des inégalités économiques entre enfants de par le monde et interprète l'ensemble de ces faits comme rendant impossible de conserver une conception «moderne» et globalisante-généralisante de l'enfance: «Une question-clé est donc de se demander dans et à travers quel réseau une forme particulière d'enfance ou d'enfant est produite» ${ }^{27}$.

Selon le sociologue appliquant le principe de symétrie, l'opposition adulte/enfant constitue un schème explicatif obsolète au regard de la réalité, fragmentée à l'extrême, des expériences enfantines et adultes dans le monde contemporain. C'est à la théorie sociologique de l'acteur-réseau que Prout emprunte son modèle pour penser l'enfance dans un univers désormais caractérisé par la dispersion et la fragmentation, un univers postmoderne. Dans ce modèle, où il convient de se demander aussi par quels relais, traductions et associations (entre humains et aussi avec des objets) une forme particulière

23 D., Bloor, Knowledge and Social Imagery, 2nd ed., Chicago, University of Chicago Press, 1991 [1976].

24 B. Latour, Nous n'avons jamais été modernes, Essai d'anthropologie symétrique, Paris, La Découverte, 2012 [1991].

25 Christensen and Prout «Les enfants, comme les adultes, cherchent individuellement et collectivement à faire de la cohérence et du sens à partir du monde dans lequel ils vivent» cité dans Taking a step away from Modernity: Reconsidering the New Sociology of Childhood, Global Studies of Childhood, 1 (1), 4-14, 2011. Traduction : A.-D. Robert.

26 A., Prout, The future of childhood, London, Routledge, 2005.

27 A. Prout, , Taking a step away from Modernity: Reconsidering the New Sociology of Childhood, Global Studies of Childhood, 1 (1), 4-14, 2011. Traduction : A.-D. Robert. 
d'enfance ou d'enfant émerge, il n'y a pas de différence avec l'adulte, car le même type de question se pose à propos de celui-ci. De même, au lieu que l'un soit défini par le fait d'être (au terme d'un développement achevé) et l'autre par le fait de devenir, adulte et enfant sont en réalité l'un et l'autre constamment aux prises avec des devenirs multiples, répondant à une multiplicité d'acteurs-réseaux. Le mot d'ordre «l'avenir est dans l'enfance» est selon Alan Prout dépassé, les sociologues de cette mouvance soutenant qu'il n'y a plus d'enfance, qu'il n'y a plus que des enfants, que ces enfants «ont» un avenir, qu'ils sont "en devenir» mais en un sens différent du sens habituel, c'est-à-dire au même titre que les adultes, comme eux impliqués dans une multitude de réseaux complexes et cloisonnés.

\section{L'ENFANT ET LA QUESTION DE L'ART}

\subsection{DE “L'ENFANT ARTISTE" À “L'ENFANT AUPRÈs DE L'ARTISTE"}

A notre connaissance, les nouvelles sociologies de l'enfance ne se sont guère intéressées à une conjoncture contemporaine dans laquelle la figure et le statut de l'enfant sont très significativement mis en mouvement, et même déplacés: l'alliance de l'enfance et de l'art ${ }^{28}$. On peut s'en étonner, et d'autant plus que ce désintérêt conduit les tenants de la sociologie postmoderne à appuyer leur conception et leur théorisation sur des exemples dramatiques que l'on peut juger à plusieurs égards discutables ${ }^{29}$. A ces exemples tout négatifs n'est-on pas fondé à opposer qu'il s'agit là non d'une illustration de la non différence entre enfants et adultes, mais bel et bien les exemples extrêmes d'une enfance bafouée, d'une enfance privée de son enfance, privée par le monde des adultes du droit à l'enfance, et donc a contrario la démonstration de la spécificité et de la différence de l'enfance?

Une illustration d'ordre positif serait autrement convaincante. C'est précisément cette illustration positive que peut fournir le domaine artistique. En effet, sous l'étiquette un peu trop lourde de «résidence artistique en milieu scolaire» ou en «milieu éducatif», depuis une bonne trentaine d'années, en France mais aussi dans de nombreux pays du monde occidental, une institutionnalisation très singulière de la rencontre entre l'art et l'enfance, mieux encore entre l'artiste et l'enfant comme personnes, est en cours. Pour désigner cette singularité, et la singularité de cette nouvelle figure de l'enfance qui s’y dessine, nous parlerons de la figure de «l'enfant auprès de l'artiste». En effet, selon

$\overline{28}$ Jean-Claude Quentel, qui n'est pas sociologue, mais psychologue, un psychologue remettant en cause la main mise de la psychologie développementale sur l'enfance, et qui en appelle à une approche anthropologique de l'enfance, fait toutefois place au dessin enfantin dans sa reconstruction de l'enfance.

Voir ci-dessus p. 6. 
nous, cette nouvelle conjoncture, et même, peut-on dire, cette nouvelle conjonction, ne se confond pas avec une autre conjonction, à laquelle nous devions une fameuse figure de l'enfance, qui peut-être considérée comme une sorte de classique de la modernité: la figure de l'enfant artiste. Dans une célèbre déclaration à son ami le photographe Brassaï, le peintre emblématique de la modernité, Pablo Picasso, avait, avec le panache qui le caractérise, immortalisé cette figure en affirmant : "Il m’a fallu toute une vie pour apprendre à dessiner comme les enfants» ${ }^{30}$.

Le propos de Picasso sonne avec superbe. Il n'est pas toutefois sans équivalent. Une enquête un peu systématique au sein de la modernité artistique pourrait sans doute en découvrir bien d'autres, de la même teneur, depuis l'injonction d'un plasticien comme Paul Klee engageant l'artiste à la démarche nécessaire pour accéder à ce qu'il appelait «l'esprit blanc» de l'enfant, jusqu'à la déclaration d'un Henri Pichette désignant le poète comme «l'homme de la plus longue enfance». Si ces propos célébrant la figure de l'enfant artiste sont assez courants tandis que l'art moderne advient, se développe et se pense comme tel, comme modernité et rupture, c'est bien parce que ces artistes de la modernité nouent avec l'enfance une sorte d'alliance substantielle. Tout préoccupés qu'ils étaient à élaborer un langage artistique en profonde rupture avec un académisme et ses codes dominants à bout de souffle, et désormais dépourvus de sens, tout déterminés à faire «sauter» l'Académie et l'Ecole des Beaux-Arts avec des tubes de peinture, comme y invitait le jeune Vlaminck, les artistes de la modernité ont trouvé dans des figures et des styles "hors normes», «hors codes», au moins pour un temps, des alliés stratégiques: dans la figure et le style enfantins, certes, mais aussi dans la figure et le style du "primitif», et encore du malade mental et de l'art "populaire», réunis dans le désormais institué «art brut ${ }^{31}$.

Assurément, cet enrôlement de l'enfance dans le combat de la modernité artistique aura contribué à modifier le statut et la figure de l'enfance; et sans doute cet «enrôlement» lui-même n'aurait pas été pensable, et moins encore possible, sans que la pensée de l'enfance et les pratiques éducatives à son égard ne connaissent les profondes transformations que l'on sait ${ }^{32}$ dans le mouvement de l'éducation nouvelle. Au sein de la modernité, il faut y insister, l'histoire de l'art et l'histoire de l'enfance connaissent des points de convergence dont il conviendrait de mieux prendre la mesure et apprécier les conséquences, pour l'une comme pour l'autre histoire.

$\overline{30}$ P. Picasso, in R. Penrose, Picasso, Paris, Flammarion, 1982, p. 361.

31 Cf. Emmanuel Pernoud, Linvention du dessin d'enfants en France, à l'aube des avant-gardes, Paris, Hazan, 2013.

32 Du moins comme on le sait quand on est familier de l'histoire des idées et des pratiques éducatives. La méconnaissance de ce domaine, qui est trop généralement la règle, prive l'histoire des idées -et ici même l'histoire de l'art- d'une dimension importante. 
La rencontre de l'art(tiste) et de l'enfance sur laquelle nous voudrions ici attirer l'attention est toutefois d'un ordre sensiblement différent, et marque une nouvelle étape dans l'histoire conjointe de l'art moderne et de l'enfance. Pour prendre la mesure de cette nouvelle figure de l'enfance en gestation dans la nouvelle alliance de l'art et de l'enfance -une figure plus "postmoderne» que «moderne»- il suffit peut-être de restituer le propos de Picasso dans son intégralité, et d'y faire sonner le contrepoint - pour ne pas dire le contrepied-qui accompagne l'éloge de l'enfance comme une sorte de modèle pour l'art qui vient: «Quand j'avais leur âge, je dessinais comme Raphaël, mais il m'a fallu toute un vie pour apprendre à dessiner comme eux». Oui, Picasso était d'abord un Raphaël en herbe... Pareille déclaration -même si dans l'esprit elle pourrait obtenir une forme d'approbation- a peu de chance, croyons-nous, de se trouver sous la plume ou dans le propos de ces artistes d'aujourd'hui qui œuvrent auprès de l'enfant, et y œuvrent bel et bien en tant qu'artistes. Non pas que tous ces artistes soient des adeptes de Joseph Beuys et effacent au nom de l'indistinction entre l'art et la vie toute différence de «métier» entre l'artiste et l'enfant; nombre d'entre eux sont fermes dans la pratique et le propos quand il s'agit de distinguer, au cœur comme au terme de la démarche artistique partagée, ce qui constitue la part de l'œuvre propre ${ }^{33}$. La raison est ailleurs: pour ces artistes, en-deçà des compétences de métier qui différencient l'enfant, tout enfant, de l'artiste accompli - cet enfant là fût-il génie en herbe annonciateur d'un nouveau Raphaël - il y a, sur le terrain de l'esthétique, sur le terrain commun, et commun à l'humanité tout entière, de l'esthétique, une égalité de l'enfant et de l'artiste.

\subsection{Esthétique et Égalité. "Egal à égaL"}

Du propos des artistes, en effet, comme des observations qui peuvent être menées là où ils œuvrent auprès d'enfants, émergent un constat et une formule récurrente, un leitmotiv : «'égal à égal». Dès lors que la rencontre s'effectue sur le terrain de l'art et de l'esthétique, la différence enfant/adulte ne va plus de soi. Tous le disent, à des degrés divers, et les observations confortent le propos: sur le terrain de l'art et de l'entrée dans l'œuvre de l'art, sur le terrain de l'esthétique et de la démarche artistique partagée, l'enfant et l'adulte sont comme naturellement "égaux». Mais de quelle «égalité» s’agit-il? D’une égalité se saisissant grâce à l'art et par l'art d'une commune et universelle humanité? D'une égalité d'atelier, d'une sorte de confraternité semi-professionnelle? D'un même enracinement symbolique? D’une égalité «de volonté», celle de libérer l'enfance en chacun?

$\overline{33}$ Jean-François Cavro, musicien accoutumé aux résidences auprès d'enfants, et qui fut notamment l'un des premiers artistes engagés dans le dispositif Enfance art et langages de la ville de Lyon, nous le signifiait récemment: "Pour la phase finale, il n'y a plus d'égal à égal, il y a mon savoir de compositeur, ma technique. Ce moment, je considère qu'il mappartient en tant qu'artiste. Il n'y a plus d'égal à égal quand je fais une ceuvre, je suis tout seul...» (Archives A. Kerlan). Pour le Centre Enfance art et langages: <http://www.eal.lyon.fr/enfance/>. 
Ces différentes dimensions de l'égalité coexistent en acte et en parole, dans la pratique des artistes et dans la théorisation qu'ils en font lorsqu'on les interroge, et dans des proportions différentes selon les artistes. Elles ont néanmoins un même sol: le sol même de l'esthétique. Il faut y insister: l'égalité enfant/adulte dont nous parlons ici ne procède pas directement d'une volonté ou d'un principe d'égalité, elle n'est pas l'effet direct de cette dynamique égalitaire propre à la démocratie, de cette "passion" démocratique de l'égalité qui affecterait l'ultime différence lui ayant jusqu'ici résisté, la différence enfant/adulte, comme l'analysent Marcel Gauchet ou Alain Renaut ${ }^{34}$, dans le sillage des réflexions de Tocqueville. Du moins ne s'y réduit-elle pas. Il faudrait en somme inverser la causalité: l'égalité de l'enfant et de l'artiste, telle qu'elle se révèle et s'éprouve sur ce terrain spécifique de l'art et de l'esthétique, est une égalité réelle, effective, et pas seulement de principe; elle relance dès lors la dynamique égalitaire.

Que recouvre en effet cet «égal à égal» surgi sur le terrain esthétique? Certes, l'engagement de l'artiste auprès de l'enfance, psychologiquement et biographiquement, n'est sans doute pas sans rapport avec sa relation à sa propre enfance. Ce serait toutefois une erreur que d'expliciter l'image de l'enfance chez l'artiste et la relation qu'il établit avec l'enfant, la proximité dont il fait régulièrement état, et le glissement de la proximité à l'égalité, en les rabattant sur la psychologie et la biographie. Répétons-le à nouveau, cette proximité se joue sur le plan de la démarche artistique, qu'on ne confondra pas avec l'imaginaire propre à chaque artiste. Ainsi, une plasticienne, Erutti, dont l'œuvre personnelle relève d'une forme d'art conceptuel, peut faire état d'une analogie entre la démarche artistique-toute démarche artistique- et ce qu'elle appelle «la démarche de l'enfant», c'est-à-dire l'être même de l'enfant en tant qu'être en construction, ou plus justement en création ${ }^{35}$.

Bien sûr, les artistes intervenants, selon qu'ils s'adressent à des enfants d'une école maternelle où à des élèves d'une classe de danse de l'Opéra de Paris (comme le musicien Jean-François Estagier), des élèves de Sciences Po (comme la chorégraphe Anne Lopez), ou à des étudiants de l'université Lyon 2 (comme le sculpteur Yves Henri) ont bien conscience des "différences» entre ces publics: mais ces différences ne sont pas premières, constitutives, dans leur engagement éducatif, dans leur démarche d'artistes intervenants. Bien des artistes intervenants le disent (et le mettent en œuvre) très clairement, même là où les «différences» pourraient sembler déterminantes: qu'il s'agisse d'enfants autistes (comme s'y consacre également Jean-François Estagier), ou de patients Alzheimer (que la chorégraphe Anne Lopez fait entrer dans la danse).

34 Renaut A., La libération des enfants. Contribution philosophique à une histoire de l'enfance, Paris, Bayard Calmann Levy, 2002; Gauchet M., "La redéfinition des âges de la vie», in L'enfant-problème, Revue Le débat, $\mathrm{n}^{\circ}$ 132, nov-déc. 2004.

35 Kerlan A. et Erruti R., Des artistes à la maternelle. Quels effets dans l'école, dans les apprentissages et le développement des enfants? Evaluer les effets de l'éducation artistique et culturelle. Symposium européen et international de recherche. Paris: Centre Pompidou/La documentation française, 2008. 
Pour s'en tenir au domaine éducatif et à l'enfance, l'intervention de l'artiste, si elle n'ignore pas l'âge des publics, ne se définit pas, ne se pense pas, en tant qu'intervention artistique, selon l'âge de ces publics. La plupart des artistes affirment que leur démarche sera la même quel que soit l'âge des enfants concernés. Voici une petite scène à laquelle tout observateur du travail de l'artiste auprès d'enfants aura très certainement assisté. Les enfants sont à l'œuvre dans la démarche que l'artiste a initiée. Les éléments plastiques s'organisent. L'artiste est un peu en retrait. Mais voilà qu'il s'approche d'un petit groupe d'enfants, qu'il s'intéresse de plus près à leurs propositions et à leurs essais. Et puis le voilà qui s'accroupit un peu plus loin, ramasse quelques morceaux de bois et de cartons, et s'essaie "pour lui-même» à une mise en forme plastique inspirée de la proposition des enfants! Un instant avant, il était en surplomb, au-dessus du groupe, en position magistrale; le voilà à présent au-même niveau, intéressé par le travail des enfants comme il le serait par celui d'un «collègue»! A la position magistrale a succédé la relation «d'égal à égal».

Y a-t-il un autre domaine que celui de l'art et de l'esthétique où la relation enfant/adulte puisse aussi aisément et «naturellement» se déplacer d'une posture verticale à une posture horizontale? Y a-t-il un autre domaine dans lequel la relation éducative puisse ainsi se déplacer d'une autorité sans conteste -car en effet l'artiste y est bien néanmoins pleinement "un maître»- à une égalité non moins «évidente»? Il est permis d'en douter. C'est pourquoi nous en sommes venus à considérer que l'expérience des artistes en résidence constitue une sorte de «laboratoire» de la relation enfant/adulte, et partant du renouvellement de la figure et du statut de l'enfance. Un laboratoire dans lequel se réinvente une relation profondément en crise. Nous sentons bien aujourd'hui, dans un temps où la relation enfant-adulte devient de plus en plus problématique, où l'autorité et la normativité en crise sont au cœur de la question éducative et de ce qu'on appelle la "crise de l'éducation» (d'un point de vue moderne), nous sentons bien la nécessité d'une autre relation, capable d'intégrer horizontalité et verticalité. L'art vivant, dans la figure de «l'enfant auprès de l'artiste» s'avère l'un des rares domaines où la dialectique de l'identité et de la différence, de l'égalité et de la distance entre l'enfant et l'adulte, le «jeune» et l'adute, joue à plein : l'artiste y est à la fois le maître, au sens fort du terme, et l'égal, capable de s'intéresser au travail, aux propositions artistiques de l'enfant avec la considération qu'il accorderait aux travaux d'un confrère, au point quelquefois d'intégrer à son propre travail les modestes essais de l'élève (forme d'illustration du principe de symétrie). L'observation comme les témoignages des artistes en fournissent des exemples réguliers et convaincants. La rencontre de l'artiste et de l'enfant est bien l'un des lieux privilégiés de cette reconstruction, et le champ de l'art et de l'esthétique son domaine d'élection. 


\subsection{SubJectivation et NORMATivité}

Sans doute une question - et une objection possible, du même coup - brûle-t-elle les lèvres du lecteur. Pourquoi l'art? Pourquoi ce privilège accordé à l'art? Pourquoi serait-ce du côté de l'art qu'il conviendrait de regarder pour essayer de comprendre ce qu'il en est de l'enfance d'aujourd'hui? Pourquoi l'éducation se tournerait-elle aujourd'hui du côté des artistes? Posons donc explicitement la question: pourquoi des artistes, non seulement à la source de l'art-ceci est la moindre des choses-mais à la source de l'éducation? Il serait bien présomptueux de prétendre répondre à ces vastes questions. Nous risquerons tout de même une hypothèse, qui rendra ces deux champs de l'art et de l'éducation beaucoup plus proches qu'on ne les considère généralement. Si nous voulons comprendre pourquoi l'art et les artistes d'aujourd'hui sont impliqués et sollicités dans le champ éducatif, nous devons nécessairement concevoir l'existence d'un intérêt commun, et même d'un intérêt majeur. Il y a en effet dans l'art d'aujourd'hui et la démarche artistique d'aujourd'hui, qu'elle se déploie sur le plan visuel, sur le plan émotionnel, ou sur ceux de l'énergie ou de la pensée, quelque chose qui touche aux questions et aux problèmes éducatifs aujourd'hui majeurs, quelque chose qui touche «à la source de l'éducation" pour notre monde. Ces questions en commun, ces problèmes partagés, pour nous en tenir à l'essentiel, nous semblent graviter autour de deux points: 1) L'individu, le sujet: comment éduquer, former le sujet aujourd'hui? 2) La norme, la loi: comment fabriquer de la norme et de la normativité tout en invitant chaque sujet à «être lui-même»? Chacun de ces points engage la question de l'enfance, et plus largement ce que nous pouvons appeler la politique de l'enfance, dans un contexte où la modernité se voit constamment battue en brèche par l'interpellation postmoderne.

La question du sujet, et plus encore de la subjectivation est bien en effet au cœur de la problématique artistique contemporaine. Paul Valéry en fut l'un des analystes les plus lucides et les plus prémonitoires. Selon la conception de la création littéraire développée par l'auteur du Cimetière marin, de la Soirée avec Monsieur Teste, l'œuvre ultime de l'artiste, au bout du compte, n'est autre que la personne même de l'artiste, lequel, à force de construire son œuvre, finit par se construirelui-même. C'est également l'enseignement que retient aujourd'hui Joëlle Zask de sa belle étude consacrée à la formation des artistes au sein des Ecoles des Beaux-Arts ${ }^{36}$ : c'est bien la personne de l'artiste lui-même qui est la matière première de la formation singulière de l'artiste; le projet artistique de l'artiste se confond avec l'artiste lui-même. Comment ne pas avoir alors à l'esprit le fameux «devenir soi-même» nietzschéen, et le «faire œuvre de soi-même» par lequel Pestalozzi définissait le but ultime de l'éducation? Dans le même registre, Pierre Gosselin, spécialiste de l'éducation artistique, s'interrogeant sur les effets éducatifs de l'art, avance qu'on ne peut les expliquer sans en passer par «la compréhension du travail de création comme mode

$\overline{36}$ Joëlle Zask, Art et démocratie. Peuples de l'art, Paris, PUF, 2003. 
de développement de la personne», et qu'il s'agit «de comprendre comment l'éducation artistique peut contribuer au développement de l'autonomie» ${ }^{37}$.

Nos observations et nos études des résidences artistiques auprès d'enfants nous conduisent à des considérations remarquablement convergentes, et renforcent notre hypothèse. La démarche artistique partagée, y compris quand ce partage s'effectue avec de jeunes enfants, s'établit de sujet à sujet. Dans sa nature même - celle d'une relation spécifiquement esthétique au monde - elle conduit à substituer, à la relation verticale de maître à élève, la possibilité ou l'ouverture permanente d'une relation horizontale, d'égal à égal, une horizontalité constamment ouverte, l'horizontalité de sujet à sujet. L'enfance, telle qu'elle peut être prédéfinie selon les modalités dominantes de la relation enfant/ adulte et les savoirs psychopédagogiques qui en décrivent le développement et les étapes, s'efface, dans la visée d'une subjectivité en partage. La relation artiste/enfant (élève) y est une relation éminemment individualisante, ou plus précisément individuante, une relation d'individuation -et ceci non pas en vertu de la "psychologie» de l'artiste, ou de sa "volonté», ou encore de son "passé scolaire», mais bien en raison de la nature même de l'art et du travail artistique. Quand par exemple la chorégraphe Anne Lopez, en résidence dans une classe artistique expérimentale de Montpellier ${ }^{38}$, émet à l'intention de tel ou tel enfant, comme le font souvent les artistes, l'adresse suivante: «Tu cherches en toi-même», il s'agit bien d'une adresse individuante, au centre de la dynamique relationnelle, parce qu' elle est au centre du travail artistique en tant que tel, et d'une adresse dont les effets pourront s'exercer certes d'abord dans l'ordre de ce qu'il est convenu d'appeler le «développement personnel», mais qui peut avoir aussi une visée ou un effet nettement «socialisant» ("la règle juste dépend aussi de toi, tu contribues à l'établissement des règles») ou encore d'ordre cognitif (appel à «'intériorisation», à la réflexivité, etc.). Le dépouillement des enregistrements filmés permet de repérer de façon précise et fine ces diverses sollicitations ${ }^{39}$, et démontre empiriquement que ce que nous appellerons l'institutionnalisation subjective est au cœur du travail artistique comme travail éducatif, formateur. Cette considération confirme la caractéristique majeure de l'expérience esthétique telle qu'elle peut être analysée sur le plan philosophique -une expérience indissociablement sensorielle, émotionnelle et intellectuelle.

37 Cf. Pierre Gosselin, «Des repères pour l'appréciation des effets de l'éducation artistique», in Evaluer les effets de l'éducation artistique et culturelle, Paris, La documentation française/Centre Pompidou, 2008, pp. 255-263.

38 Le collège Les escholiers de la Mosson est le lieu d'une expérimentation pédagogique en cours. De la classe de sixième à la classe de troisième - les élèves sont, au moment où nous rédigeons cet article, en classe de quatrième - une même classe bénéficie l'année entière d'une résidence artistique à raison de six heures hebdomadaires. A une compagnie chorégraphique la première année a succédé en classe de cinquième une double résidence: une compagnie théâtrale et un écrivain. L'année en cours accueille un groupe musical et le même écrivain.

39 Ces analyses font l'objet d'une thèse de doctorat en cours (Céline Choquet, direction Alain Kerlan, université Lumière Lyon2, laboratoire éducation Cultures, Politiques). 
De surcroît, dans la démarche artistique partagée entre l'enfant et l'artiste, ces deux problématiques contemporaines communes à l'art et à l'éducation d'aujourd'hui, celle du sujet et celle de la norme, s'avèrent articulées l'une à l'autre. L'adresse subjectivante, individuante, que nous avons relevé dans le travail d'Anne Lopez avec des élèves de collège, sur l'un de ses versants faisait déjà signe vers le champ des règles. Le domaine des pratiques artistiques comme espace d'une autre rencontre éducative est précisément l'un des lieux contemporains où cette action des normes et la fabrique des sujets peuvent être interrogés ${ }^{40}$; où peuvent être saisies conjointement la fabrique des sujets et la fabrique des normes. De nombreuses observations pourraient l'illustrer. Dans chaque situation de démarche artistique partagée entre l'artiste et les enfants, viennent ces moments où l'enfant se tourne vers l'artiste, et l'interroge: «Est-ce que cela va comme ça? Suis-je sur la bonne voie? Et qu'est-ce que je fais maintenant?» Etc. La demande est claire: il s'agit de s'enquérir de la conformité du travail à un modèle, à une vérité, à une norme dont l'artiste est supposé détenir la clé. Et la réponse de l'artiste est invariablement la même: "Cherche, la solution, la réponse à ta recherche n'est pas à l'extérieur de toi, mais elle est en toi, en toi et du côté de cette norme immanente à laquelle ton travail obéit et qu’il faut que tu découvres». Ne confondons pas cette injonction avec l'une de ces formes d'un socratisme de façade régnant dans le monde scolaire. Dans le monde scolaire traditionnel, précisément, ce monde scolaire précisément en crise, les normes et règles sont pour l'essentiel de l'ordre d'un déjà-là. Il en va autrement selon l'ordre propre au travail artistique. Le travail artistique procède d'une autre normativité, d'une normativité proprement immanente. Interrogé sur le rôle de l'art et de l'artiste auprès d'enfants, sur leur apport spécifique, le peintre Gérard Garouste fait cette réponse: «Ce que l'artiste apporte d'abord? Une nécessaire et salutaire déstabilisation... Ce n'est pas l'absence de normes, mais la capacité à produire, travailler, déplacer la normativité qui importe...»" ${ }^{41}$. Gérard Garouste démonte ainsi le lieu commun qui fait de l'art en éducation une donation de sens et de normes, de valeurs, dans un monde qui en serait dépourvu, un recours stabilisateur pour un monde instable. Avec l'art et l'artiste, à l'inverse, dans le partage artistique, il s'agit de la capacité à refuser la fermeture du monde sur un sens donné, un sens tout fait, bouclé, sur un monde sans fenêtre. Mais ce refus n'est pas refus de la norme et de la loi. La normativité immanente à l'œuvre et en partage dans le travail de l'art renvoie à une conception de la norme en résonance avec le sujet contemporain, lequel ne peut plus exister sous une unique norme en surplomb. En résonance donc avec les exigences de la démocratie contemporaine. On l'oublie trop souvent: le travail artistique

$\overline{40}$ C'était, comme on le sait, la préoccupation première de Michel Foucault: comprendre comment l'action des normes dans la vie des hommes détermine le type de société auquel ceux-ci appartiennent comme sujets.

41 G. Garouste, in Alain Kerlan (dir.), Des artistes à la maternelle, Scéren/CNDP 2005. Entretien repris dans la revue en ligne Sens Public, et accessible sur son site: <http://www.sens-public.org/ spip.php?article894>. 
est nécessairement affrontement à la norme et à la loi, celles-là même de l'œuvre, celles-là même sans lesquelles l'œuvre ne pourrait advenir.

\section{CONCLUSION}

Nous voici, à cette étape de la réflexion, en présence d'un singulier renversement. Nous inscrivant dans le sillage des analyses de Marcel Gauchet et d'Alain Renaut, et ne dissociant donc pas l'avènement des nouvelles figures de l'enfance des avancées et exigences de la démocratie, nous ne nous étonnerons pas que la figure de «l'enfant auprès de l'artiste» les concerne au plus vif. Si la passion de l'égalité conduit à l'effacement de l'ultime différence, la différence entre l'enfant et adulte, il y a bien des raisons pour que l'art soit l'un des terrains où s'essaient cet effacement et la recomposition relationnelle qu'il implique. La figure "moderne» de «l'enfant artiste» participait sans doute de la dynamique égalitaire, mais ne remettait pas en cause le développementalisme; ou plus précisément, dans le temps même où la figure de l'enfant artiste commençait à brouiller les frontières, le développementalisme les diffractait en autant d'étapes. Il en va différemment de la figure de l'enfant auprès de l'artiste: il s'agit d'une figure résolument post-développementaliste, que l'on peut bien dire, à ce titre, postmoderne. Les espaces que ménagent au sein du champ éducatif les résidences artistiques auprès d'enfants peuvent alors être regardés comme les «laboratoires» de l'individualisme démocratique, les laboratoires d'une postmodernité démocratique en gestation, où s'essaie et s'invente une autre relation éducative, une autre relation adulte/enfant, une relation dans laquelle l'alternance, la dialectique de l'horizontalité et de la verticalité seraient possibles, dans laquelle l'égalité de l'enfant et de l'adulte, nouvelle étape de la passion égalitaire inhérente à la démocratie, serait compatible avec une responsabilité éducative refondée. Oui, assurément, il y a tout lieu d'inciter les nouvelles sociologies de l'enfance à s'intéresser à la nouvelle alliance de l'art et de l'enfance. 